\title{
Grain Boundaries Imaged by Integration of Sobel Filtered Scanning Transmission Electron Micrographs
}

\author{
Min-Chul Kang, Jinsu Oh, Cheol-Woong Yang*
}

School of Advanced Material Science \& Engineering, Sungkyunkwan University, Suwon 16419, Korea

*Correspondence to:

Yang $\mathrm{CW}$,

(iD) http://orcid.org/0000-0003-0475-8399

Tel: $+82-31-290-7362$

Fax: +82-31-290-7371

E-mail: cwyang@skku.edu

Received December 25, 2018

Revised December 27, 2018

Accepted December 28, 2018
One of the most important factors determining the properties of a material is its grain size. However, unclear grain boundaries in the image hinder an accurate measurement of grain size. We demonstrate that grain boundaries existing in the images obtained by scanning transmission electron microscopy (STEM) can be clearly distinguished by applying a Sobel filter to a tilting series of STEM images of a hydrogenation-disproportionationdesorption-recombination processed $\mathrm{Nd} 2 \mathrm{Fe} 14 \mathrm{~B}$ magnet sample.

Keywords: Sobel filter, Image processing, Poisson noise, Channeling contrast
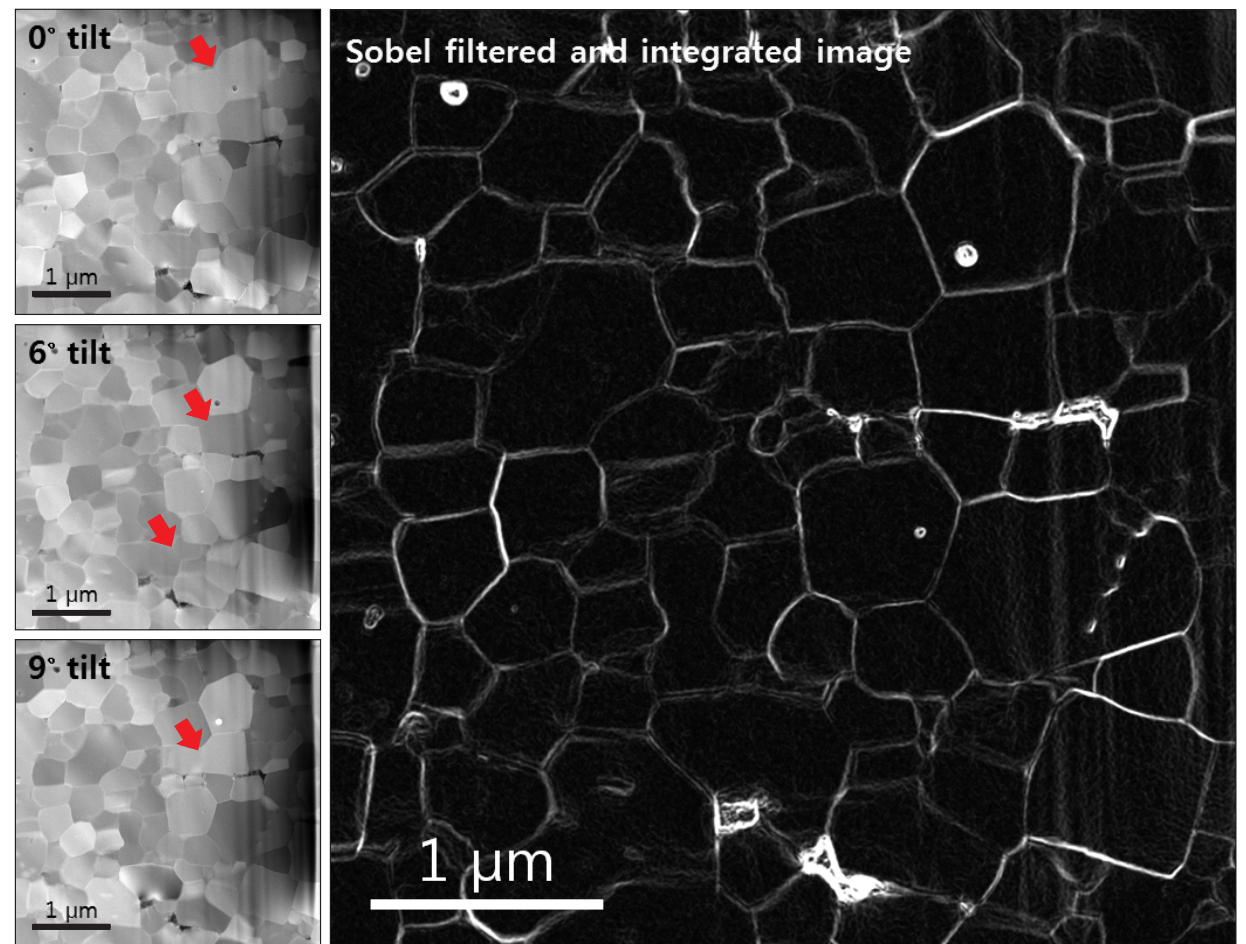

@ (T) This is an open-access article distributed under the terms of the Creative Commons Attribution Non-Commercial License (http://creativecommons.org/licenses/by-nc/4.0) which permits unrestricted noncommercial use, distribution, and reproduction in any medium, provided the original work is properly cited.

Copyrights @ 2018 by Korean Society of Microscopy 


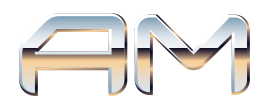

Generally, the grain size has a great influence on the physical properties of a material (Pauli et al., 2014). To accurately measure the grain size from the conventional images, computer process so called Sobel filter (Sobel et al., 1968) can be used. It computes gradient change between neighboring pixels and emphasizes the outline of objects.

In the case of very fine grains, a considerable high resolution is required to measure the grain size. Therefore, transmission electron microscopy (TEM) and/or scanning transmission electron microscopy (STEM) observation is necessary. It may be preferred to use bright field (BF)-STEM because pure channeling contrast that is strongly related to the grain orientation can be easily extracted through BF-STEM imaging. For some samples such as magnetic materials, other contrast (such as Lorentz contrast) can be mixed in conventional TEM image. Besides, although the orientation of neighboring grains is different each other, the channeling contrast can be almost the same when the lattice structure (configuration of atomic column) of the adjacent grains is similar with respect to the beam direction, and thus grain boundary becomes unclear as indicated with red arrows in the figures. In order to distinguish the grains having similar channeling contrast, a series of BF-STEM images was acquired tilting the sample at various angles as shown on the left hand side of the image. These images were obtained from a HDDR (hydrogenation-disproportionationdesorption-recombination) processed $\mathrm{Nd}_{2} \mathrm{Fe}_{14} \mathrm{~B}$ magnet sample using aberration corrected STEM (JEOL ARM-200F). After then, Sobel filter was applied to each image to compute the gradient and integrated. A noise induced by digital signal acquisition (Poisson noise) makes the gradient value unstable. To minimize this effect, images were recorded at slow scan speed and with high probe current to get enough electron dose. Distortion induced by tilt was corrected by numerical geometric calculation. This process can make it possible to distinguish the grains in nearby region which cannot be easily distinguished each other.

\section{CONFLICT OF INTEREST}

No potential conflict of interest relevant to this article was reported.

\section{ACKNOWLEDGMENTS}

This work was supported by the National Research Foundation of Korea (NRF) grant funded by the Korea government (MSIP) (2018R1A5A6075959).

\section{REFERENCES}

Pauli L, Heikki R, Tapio S, Hannu H, Jani R (2014) Influence of grain size distribution on the Hall-Petch relationship of welded structural steel. Materials Science \& Engineering A. 592, 28-39.
Sobel I, Feldman G (1968) A 3×3 isotropic gradient operator for image processing. a talk at the Stanford Artificial Project. 